\title{
First Results from the Axion Dark Matter eXperiment (ADMX) Upgrade
}

\author{
Karl Van Bibber* \\ Lawrence Livermore National Laboratory \\ E-mail: vanbibber1@llnl.gov

\section{Gianpaolo Carosi} \\ Lawrence Livermore National Laboratory \\ E-mail: carosi2@llnl.gov
}

\begin{abstract}
The Axion Dark Matter eXperiment (ADMX) searches for axions which may constitute some or all of the dark matter of the halo of our Milky Way galaxy. The experiment relies on the principle that, through the Primakoff effect, an axion can resonantly convert into a single monochromatic photon in a microwave cavity permeated by a magnetic field. In its first eight years of operation, AMDX set limits on dark matter axions in the $m e V$ range for important classes of models. Recently the experiment has been upgraded to utilize newly-developed SQUID amplifiers which promise much lower equivalent noise temperatures, and first results are presented here.
\end{abstract}

Identification of dark matter 2008

August 18-22, 2008

Stockholm, Sweden

${ }^{*}$ Speaker. 


\section{Introduction}

Axions result from the most minimal and elegant solution to the strong-CP problem, i.e. why the neutron has no electric dipole moment $[1,2]$. The axion is a pseudoscalar, i.e. $J^{\pi}-0^{-}$, and thus can couple to two photons like the neutral pion. Its particle physics properties have been discussed thoroughly in the report of Kim at this conference [3]; here we only express the mass of the axion and its two-photon coupling in terms of the Peccei-Quinn symmetry-breaking scale $f_{a}$, the single major parameter of the theory:

$$
\begin{gathered}
m_{a} \simeq 0.6 \mathrm{eV} \frac{10^{7} \mathrm{GeV}}{f_{a}} . \\
g_{a \gamma \gamma}=\frac{\alpha g_{\gamma}}{\pi f_{a}}
\end{gathered}
$$

In the axion-photon coupling above, $g_{\gamma}$ is a dimensionless model-dependent parameter of order 0.1-10. Two well-developed classes of models are those of Kim, Shifman, Vainshtein and Zakharov, or KSVZ [4,5] where the axion couples only to quarks and possibly only to very heavy quarks, and the more generic GUT-inspired model of Dine, Fischler, Sreknicki and Zhitnitskii, or DFSZ [6, 7].

Cosmology and astrophysics constrain the axion's mass and coupling. The fraction of the energy density of the universe in axions, $\Omega_{a} \approx 6 \mu \mathrm{eV} / \mathrm{m}_{a}$, means that axions much lighter than the microelectronvolt range pose a severe overclosure problem for the universe. In fact, the energy density associated with all matter, both the dominant dark and subdominant baryonic component total to $\Omega_{m} \approx 0.27$, which would imply a stricter lower limit on the axion mass, but there are significant uncertainties associated with the predicted cosmological abundance of axions above. Stellar evolution also provide rigorous constraints on axions; the general argument is that axions produced in stellar interiors and free-streaming out of stars would represent an energy-loss channel which would disrupt the agreement between astronomical observations and stellar evolution calculations. For example, for the Type-II supernova SN1987a, axions heavier than $\approx 1 \mathrm{meV}$ would have been produced copiously, free-streaming from the proto-neutron core, and would have foreshortened the neutrino pulse observed in both the IMB and Kamioka neutrino detectors; and are thus excluded. This limit ceases to be valid for axions of mass $>\mathrm{O}(1 \mathrm{eV})$, but other limits take over for heavier axions. Finally, systematics of cohort populations of Horizontal Branch stars preclude axions with radiative coupling of $g_{a \gamma \gamma}>10^{-10} \mathrm{GeV}^{-1}$. Thus nominally, the search window for axions is $10^{-6}<m_{a}<10^{-3} \mathrm{eV}$, with lower masses favored if the axion is to be the dominant form of dark matter in the universe.

\section{The microwave cavity experiment}

In principle, the axion could decay into two photons, but the spontaneous decay lifetime, which goes as $t \propto m_{a}^{-3} \cdot g_{a \gamma \gamma}^{-2} \propto m_{a}^{-5}$, vastly exceeds the age of the universe for axions in the allowed mass range. Such light axions are essentially stable cosmologically, and had been dubbed 'invisible axions' due to their miniscule couplings. However, pseudoscalars can undergo a Primakoff interaction, meaning that an axion in an external electromagnetic field (a sea of virtual photons) 
can convert into a single real photon with the full energy (mass + kinetic) of the particle; likewise for the inverse process. The Lagrangian density which determines the interaction is given by

$$
L_{a \gamma \gamma}=-\left(\frac{\alpha}{\pi} \frac{g_{\gamma}}{f_{a}}\right) a \mathbf{E} \cdot \mathbf{B}
$$

In the case of the microwave cavity experiment to be described, $a$ is the axion field, $B$ the external magnetic field, and $E$ the electromagnetic field associated with the converted photon.

In 1983 Pierre Sikivie published a concept for an axion search based on the Primakoff interaction, which would render their discovery feasible within the constraints of current technology $[8,9]$. In this scheme, axions resonantly convert to microwave photons in a high-Q microwave cavity permeated by a strong magnetic field (Figure 1 ). The cavity is tunable, as the resonant conversion condition requires $h v=m_{a} c^{2}\left(1+\frac{1}{2} \beta^{2}\right) \approx m_{a} c^{2}\left(1+O\left(10^{-6}\right)\right)$, where $v$ is the cavity frequency. The $T M_{010}$ mode is generally used as it has the highest overlap, or 'form factor' of the electromagnetic mode and the uniform magnetic field.

The signal power due to axion-photon conversion is given by

$$
P_{a \rightarrow \gamma} \propto\left(B_{0}^{2} V Q\right) \cdot\left(g_{\gamma}^{2} \frac{\rho_{a}}{m_{a}}\right)
$$

At each central frequency of the cavity mode, the power spectrum is calculated by a Fast Fourier Transform within a narrow frequency interval determined by the bandpass of the cavity, $\Delta v \sim v / Q$, where $Q$ is the quality factor of the cavity mode, typically of order $10^{(4-5)}$. After each run of $\sim 100$ seconds, the central cavity frequency is incremented by a small amount; the final power spectrum results by an optimally-weighted co-addition of the overlapping individual runs. Two spectra are actually calculated; a medium-resolution channel with binning $\Delta E / E \sim$ $10^{-6}$, well-matched to the expected thermalized component of the axionic dark matter, and a highresolution channel $\Delta E / E \sim 10^{-11}$, to search for fine-structure associated with late-infall axions predicted within several models [24, 25]

\section{The Axion Dark Matter eXperiment (ADMX)}

Two liter-scale microwave experiments were performed in the late 1980s and early 1990s; one a Rochester-Brookhaven-Fermilab (RBF) collaboration [10,11, 12], and the other at the University of Florida (UF) [13]. A great deal was learned about the optimal technical approach, design details and search strategy, and these experiments came within two orders of magnitude in $g_{a \gamma \gamma}$ of plausible axion models.

In 1993, the ADMX collaboration formed to build a second-generation experiment, drawing from the experience of the RBF and UF experiments, with the goal to achieve the sensitivity required to find or exclude QCD axions at a practical search rate ${ }^{1}$. The experiment would achieve the required sensitivity by both a large scale-up in volume, and capitalizing on the steady improvements in the noise performance of the state-of-the-art microwave amplifiers. The magnet is a superconducting solenoid $(\mathrm{NbTi})$ of $1.1 \mathrm{~m} \times 0.6 \mathrm{~m}$ radius, with a maximum field on axis of 8.5 tesla, which

\footnotetext{
${ }^{1}$ At present the ADMX collaboration consists of the University of Florida, Lawrence Livermore National Laboratory, the National Radio Astronomical Observatory and the University of Washington.
} 


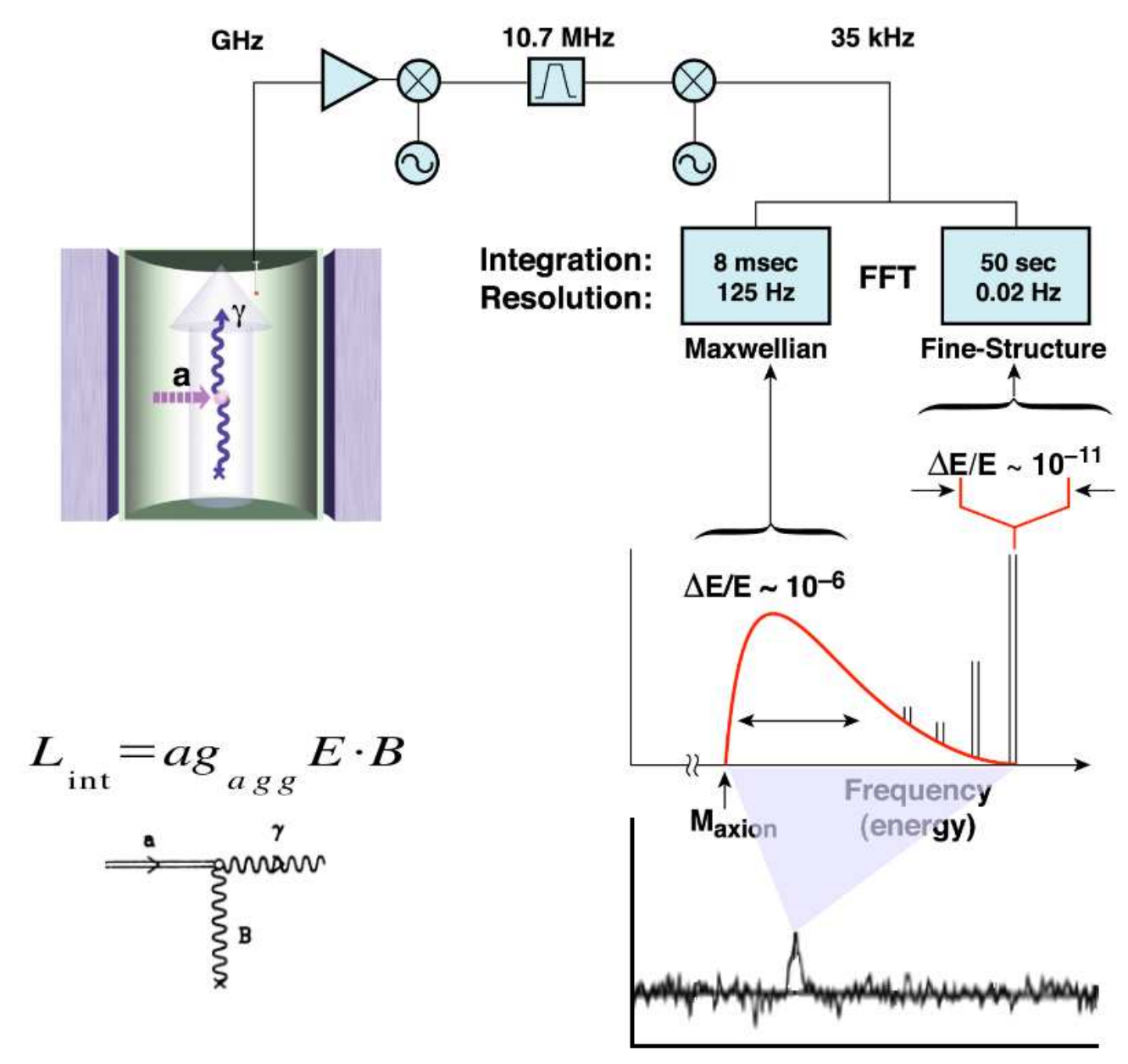

Figure 1: (Left) The Primakoff interaction by which an axion couples to a virtual photon (an external magnetic field) and a single real photon carrying its full energy. (Right) Schematic of the microwave cavity experiment. The experiment is a double-heterodyne receiver by which gigahertz frequencies are mixed down to the kilohertz range, and the resulting power spectra calculated by a Fast Fourier Transform. In the Axion Dark Matter eXperiment (ADMX) the signal bifurcates into a medium-resolution channel, optimized to search for the thermalized component of axionic halo dark matter, and a high-resolution $\left(\Delta E / E \sim 10^{-11}\right)$ channel, to look for any fine-structure of the signal, as has been predicted in numerous studies.

accomodates a high-purity annealed copper cavity of $1.0 \mathrm{~m} \times 0.5 \mathrm{~m}$ radius. The series of Heterojunction Field-Effect Transistor (HFET) amplifiers utilized in the original ADMX experiment were designed and fabricated by NRAO, and achieved an equivalent noise temperature of $T_{N} \sim 1.5 \mathrm{~K}$ in the gigahertz range [14]. The physical temperature of the experiment (i.e. the cavity blackbody temperature) was $T \sim 1.5 K$, achieved by Joule-Thompson cooling, thus rendering the total system noise to be $T_{S}=T+T_{N} \sim 3 K$ at best. Figure 2 (left) shows the ADMX cryogenic tower just after being withdrawn from the magnet cryostat.

In its original design, ADMX operated successfully from early 1996 until 2003, at which time it was shut down in order to upgrade to the newly-developed near-quantum-limited SQUID amplifiers. During the initial data run, axions were excluded as halo dark matter for axion-photon 

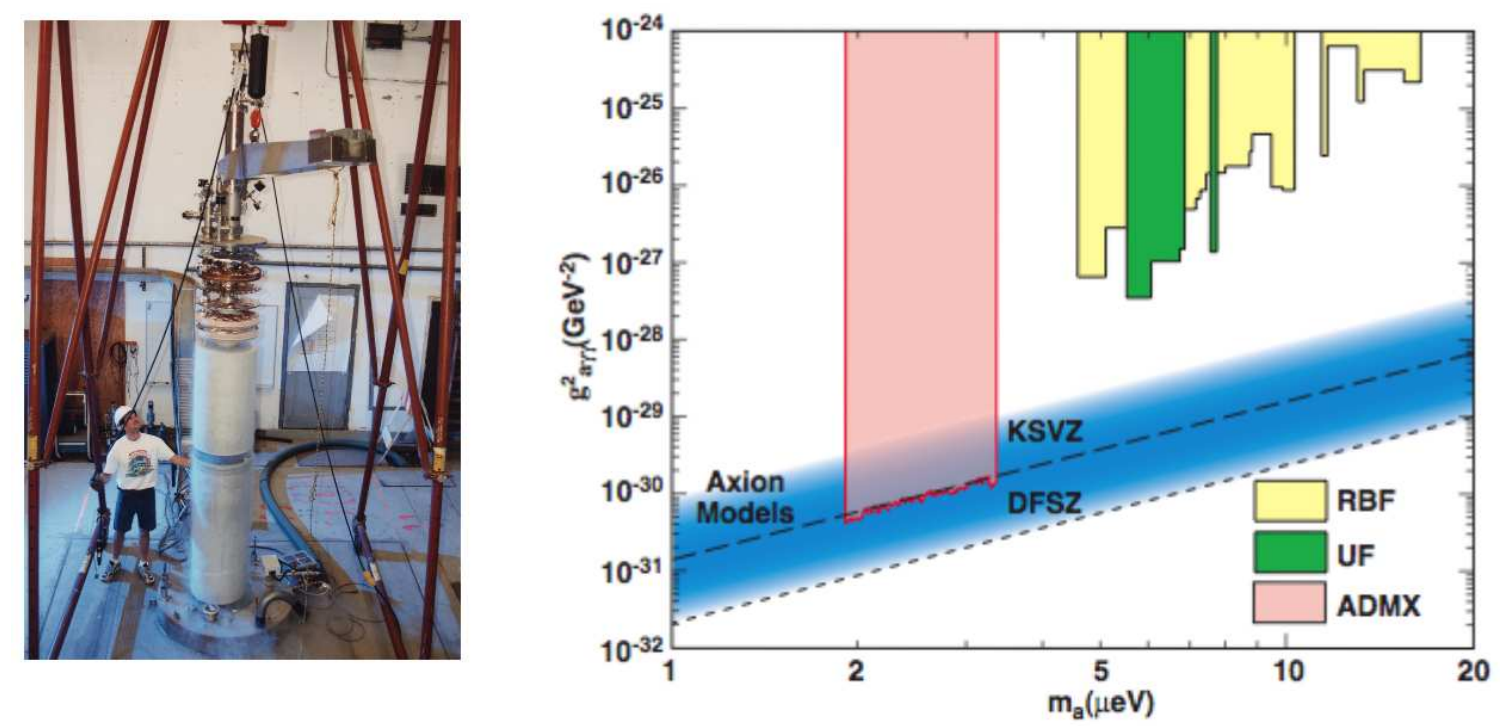

Figure 2: (Left) Photograph of the ADMX cryogenic tower after being withdrawn from the magnet. The microwave cavity is housed within the vacuum shroud at the bottom of the insert; just below its LHe cryostat. The top flange of the magnet cryostat is visible at floor level; the superconducting coil itself is located 3 meters below. (Right) The region in axion mass and photon coupling excluded in the first ADMX running period. Also shown are the exclusion regions from the pilot experiments at Brookhaven (RBF) $[10,11,12]$, and the University of Florida (UF) [13].

couplings in the middle of the model region, over approximately an octave of mass [Figure 2 (right)] $[16,17,18,19,20]$. Furthermore, the experiment demonstrated the greatly increased sensitivity associated with the high-resolution search [19, 20], as well as optimized filters [18]. A complete description of the experiment and analysis procedure is found in [21]. No axion was found.

\section{Upgrade to SQUID amplifiers}

Superconducting QUantum Interference Devices (or SQUIDs), exploiting the unusual V-I characteristics of Josephson junctions, are exquisitely sensitive flux-to-voltage transducers, which have been long employed as magnetometers and low-frequency amplifiers. Motivated by the needs of the dark-matter axion search, John Clarke and his group at UC Berkeley developed a microstripcoupled SQUID which performed as a near-quantum-limited amplifier well into the gigahertz regime $[22,23]$. A schematic and microphotograph of a microstrip-coupled SQUID amplifier are shown in Figure 3 (left). Unlike conventional microwave amplifiers, the SQUID intrinsic noise performance continues to improve linearly as the physical temperature is decreased; the linearly decreasing noise reflects the Johnson noise of the shunt resistors in parallel with the Josephson junctions. Ultimately the noise performance bottoms out for reasons that are not fully understood, but are generally ascribed to athermal electron effects; the best SQUID amplifiers to date achieve noise temperatures only about $15 \%$ above the standard quantum limit.

The microstrip-coupled SQUID amplifiers have been made with gains of up to $30 \mathrm{~dB}$, operating at frequencies as high as $3 \mathrm{GHz}$. Another important feature of the design is the ability to 

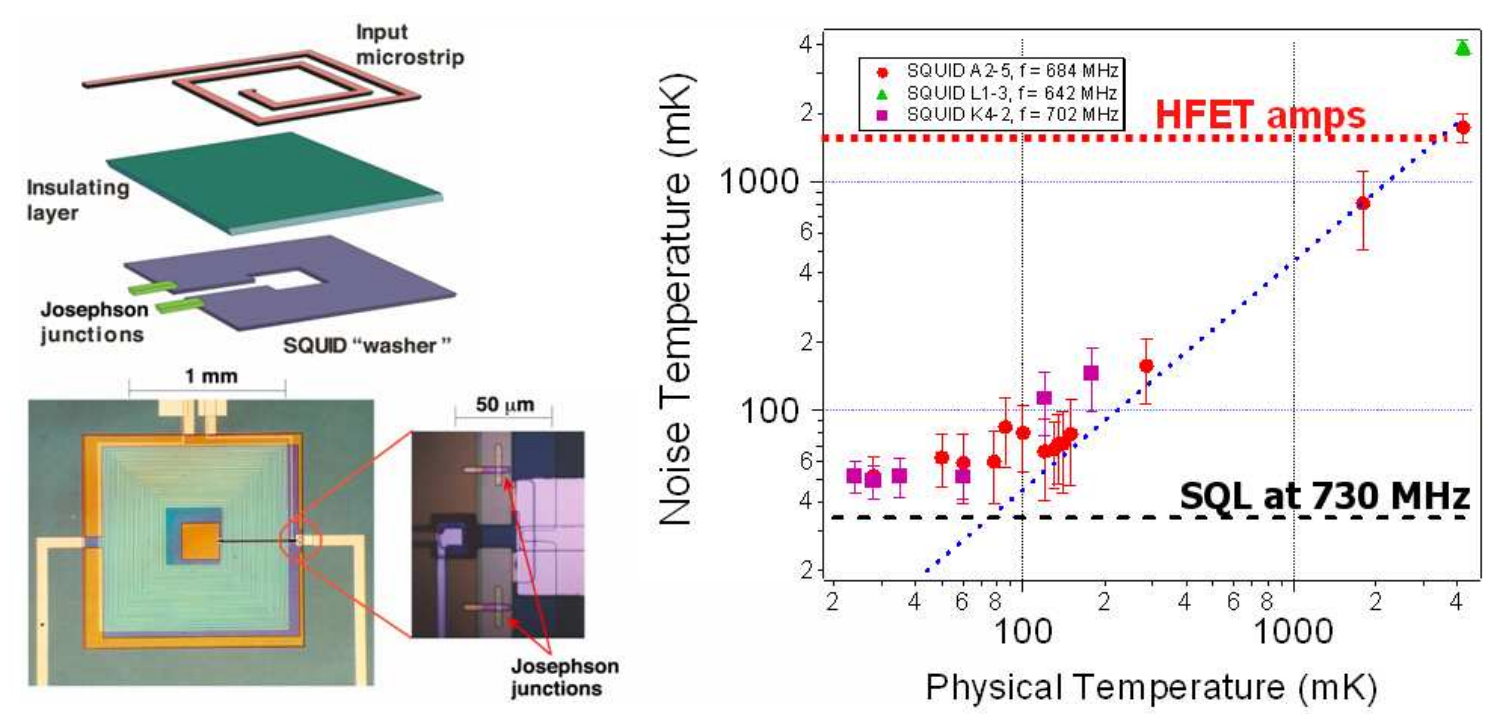

Figure 3: (Left, Top) Exploded schematic of the microstrip-coupled SQUID amplifier. (Left, Bottom) Microphotograph of the SQUID amplifier, with the Josephson junctions shown in the expanded view. (Right) Noise equivalent temperature of the microstrip-coupled SQUID amplifier as a function of physical temperature at a frequency of $\sim 700 \mathrm{MHz}$. The best SQUIDs achieve intrinsic noise temperatures within $15 \%$ of the Standard Quantum Limit, $k T=h v$.

tune the central frequency of the SQUID by almost a factor of two. This SQUIDs also work with reactive loads, such as microwave cavities and they can be staged to achieve greater gains [26].

\section{The Phase-I Upgrade and First Results.}

Upgrading ADMX to capitalize on this breakthrough in noise performance required a major hardware upgrade, owing to a small but significant technical difference between HFET and SQUID amplifiers. Namely, while HFET amplifiers work well within a magnetic field, so long as the trajectory of the electrons in the gate is oriented along the field direction, the SQUID amplifiers require an essentially magnetic field-free environment to operate at all. As the main magnet coil possesses an enormous fringe field, $\sim 0.5 T$ at the position of the amplifier a meter above the top of the coil, a major redesign of the experiment was necessary to incorporate an active fieldcompensation coil and passive magnetic shielding around the amplifier housing. A new vacuum and cryogenic design was also required.

It was decided to carry out the ADMX upgrade in two phases. The Phase-I upgrade, carried out between 2004 - 2007, retrofitted the experiment with SQUID amplifiers, to demonstrate that SQUID amplifiers could perform practically and smoothly in the microwave cavity experiment. However, as the physical temperature of the experiment, including the SQUIDs themselves, remains at $T \sim 2 K$, the intrinsic SQUID amplifier noise temperature is not greatly different from that of the HFETs previously used, and likewise the total system noise temperature remains as before, $T_{S} \sim 3 K$. After a year of data-taking, the Phase-II upgrade will begin, adding a dilution refrigerator to reduce the physical temperature of the experiment to $T \sim 100 \mathrm{mK}$, where the intrinsic SQUID performance is best, leading to a total system noise temperature $T_{S} \sim 200 \mathrm{mK}$. This will allow 
the mass range to be scanned at several times the current rate, probing the most pessimistic axion models, even if axions only constitute a minority fraction of the halo.

Figure 4 shows the first small window of mass range to be covered by the Phase-I upgrade, after recommissioning in late-Spring 2008. Consistent with the previous data, the experiment readily achieved sensitivity to axion-photon couplings in the middle of the band of models explored theoretically.

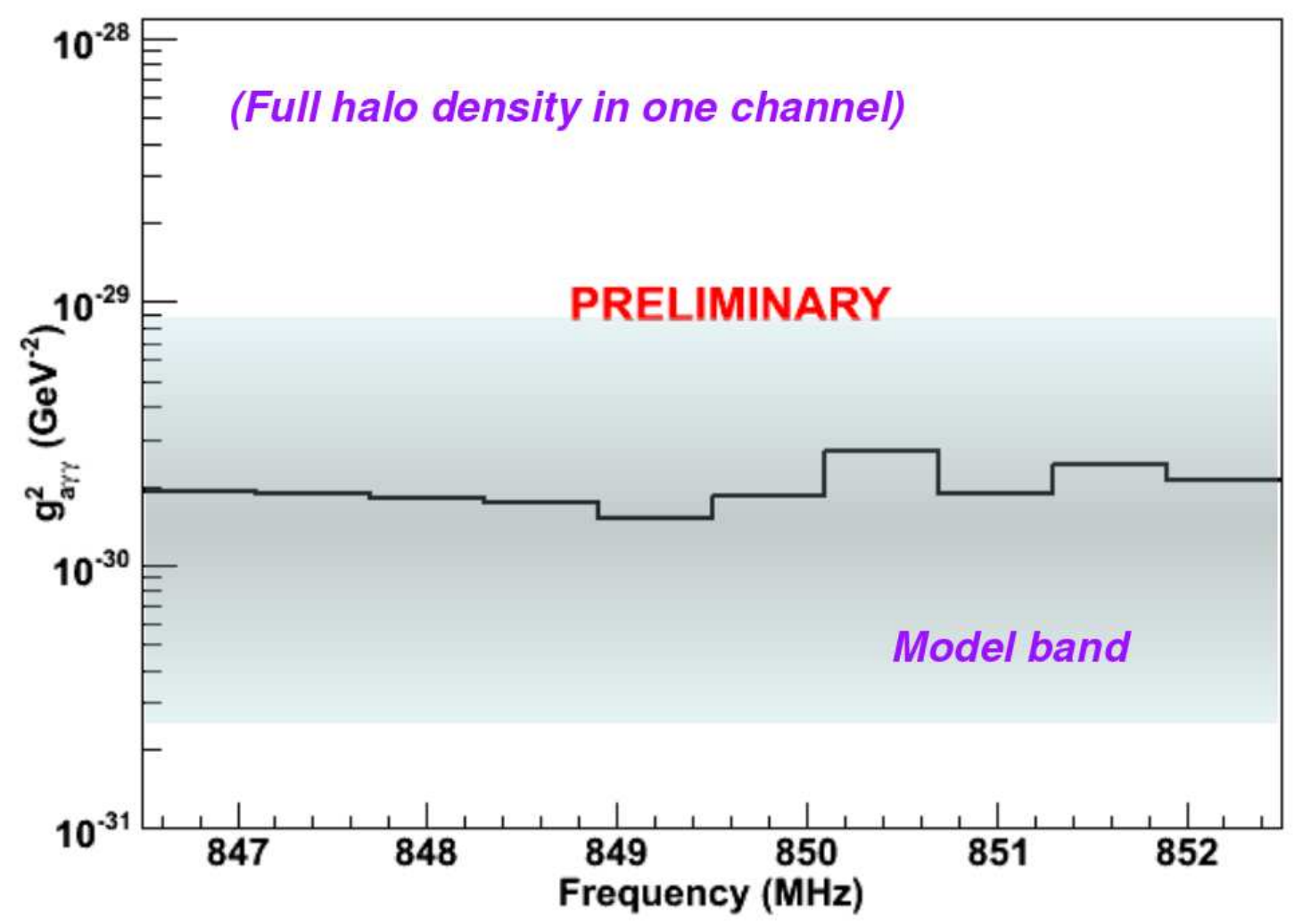

Figure 4: Axion-photon coupling vs. mass, in a narrow range of mass around $m_{a} \sim 3.5 \mathrm{meV}$, from the initial data of the ADMX Phase-I upgrade to SQUID amplifiers (preliminary).

This work was supported in part by the U.S. Department of Energy under Contract No. DE AC52-07NA27344. The support of the Laboratory Directed Research and Development Program for enabling technology development is gratefully acknowledged.

\section{References}

[1] R.D. Peccei and H.R. Quinn, Phys. Rev. Lett., 38, 1440-1443, 1977.

[2] S. Weinberg, Phys. Rev. Lett, 40, 223-226, (1978).

[3] J.E. Kim, IDM2008 conference, Aug. 22, 2008.

[4] J.E. Kim, Phys. Rev. Lett., 43, 103-107, (1979).

[5] M.A. Shifman, A.I. Vainshtein and V.I. Zakharov, Nuc. Phys. B, 166, 493-506, (1980). 
[6] A.R. Zhitnitshii, Sov. J. of Nucl. Phys., 31, 260, (1980).

[7] M. Dine, W. Fischler and M. Srednicki, Phys. Lett. B, 104, 199-202, (1981).

[8] P. Sikivie, Phys. Rev. Lett., 51, 1415-1417, (1983).

[9] P. Sikivie, Phys. Rev. D, 32, 2988-2991, (1985).

[10] S. DePanfilis et al., Phys. Rev. Lett., 59, 839-842, (1987).

[11] Y. Semertzidis et al., Phys. Rev. Lett., 64, 2988-2991, (1990).

[12] R. Cameron et al., Phys. Rev. D, 47, 3707-3725, (1993).

[13] C. Hagmann et al., Phys. Rev. D, 42, 1297-1300, (1990).

[14] R. Bradley et al., Rev. of Mod. Phys., 75, 777-817, (2003).

[15] C. Hagmann et al., Phys. Rev. Lett., 80, 2043, (1998).

[16] S. Asztalos et al., Phys. Rev. D, 64, 092003, (2001).

[17] S. Asztalos et al., ApJ, 571, L27-L30, (2002).

[18] S.J. Asztalos et al., Phys. Rev. D, 69, 011101, (2004).

[19] L. Duffy et al., Phys. Rev. Lett., 95, 091304, (2005).

[20] L.D. Duffy et al., Phys. Rev. D, 74, 012006, (2006).

[21] S. Asztalos et al., Nucl. Instr. and Meth. A, 444, No. 3, 21, (2000).

[22] C. Hilbert and J. Clarke, J. of Low Temp. Phys., 61, 263-279, (1985).

[23] M. Mück, M.O. Andrĕ, J. Clarke, J. Gail and C. Heiden, App. Phys. Lett., 72, 2885-2887, (1998).

[24] P. Sikivie and J.R. Ipser, Phys. Lett. B, 291, 288-292, (1992).

[25] A. Natarajan and P. Sikivie, Phys. Rev. D, 72, 083513, (2005).

[26] M. Mück, M.O. Andrĕe, J. Clarke, J. Gail and C. Heiden, App. Phys. Lett., 75, 3545, (1999). 